\title{
The hurdles of nanotoxicity in transplant nanomedicine
}

\author{
Amber Nagy*,1 \& Nicholas L Robbins ${ }^{1}$ \\ ${ }^{1}$ 59th Medical Wing, Office of Science \& Technology, Joint Base San Antonio-Lackland, TX 78236, USA \\ *Author for correspondence: amber.m.mallory2.civ@mail.mil
}

\begin{abstract}
Nanomedicine has matured significantly in the past 20 years and a number of nanoformulated therapies are cleared by regulatory agencies for use across the globe. Transplant medicine is one area that has significantly benefited from the advancement of nanomedicine in recent times. However, while nanoparticlebased therapies have improved toxicological profiles of some drugs, there are still a number of aspects regarding the biocompatibility and toxicity of nanotherapies that require further research. The goal of this article is to review toxicological profiles of immunosuppressant therapies and their conversion into nanomedicine formulations as well as introduce future challenges associated with current in vitro and in vivo toxicological models.
\end{abstract}

First draft submitted: 10 May 2019; Accepted for publication: 13 August 2019; Published online:

23 October 2019

Keywords: biocompatibility $\bullet$ immunosuppressant $\bullet$ nanomedicine $\bullet$ nanoparticle $\bullet$ sirolimus $\bullet$ tacrolimus $\bullet$ toxicity

Nanoparticles (NPs), less than $100 \mathrm{~nm}$ in one dimension, are attractive candidates for use in numerous consumer and medical applications due to their size, versatility and customizable potential [1]. Engineered NPs have proven to be effective chemotherapeutics, and multiple formulations are cleared by the US FDA mainly in the form of polymeric NPs, micelles, liposomes or nanocrystals [2]. These NPs have been cleared for treatment of cancer, rheumatoid arthritis, Crohn's disease, multiple sclerosis, hepatitis B and C, kidney disease and diabetes - among others [3]. Incorporating therapeutics into small, encapsulated forms opened up an entirely different avenue for targeted delivery, particularly for highly hydrophobic molecules, and includes immunosuppressant therapies for transplant recipients. However, with the excitement surrounding the potential for nano-based materials to push medicine into its next defined phase, the safety and risk-benefit analyses have yet to be fully investigated. There is concern that the size-based properties that make NPs so unique may also cause adverse health effects. Increasingly, regulatory agencies have placed more emphasis on understanding the safety and toxicity of NPs since the behavior of NPs in biological systems is not completely understood. Accordingly, substantial efforts have been made to determine if they behave differently from bulk material, from a toxicological perspective.

The goal of this effort is to present a background on commonly used nanotoxicity assays and results from relevant experiments assessing toxicity of immunosuppressants as nanoformulations in vitro and in vivo as they pertain to transplant medicine.

\section{Conventional nanotoxicity assay constructs: in vitro \& in vivo}

Approaches for measuring NP toxicity in vitro

The field of nanotoxicology exploded onto the scientific scene in the 1990s. Concurrent to the development of novel NPs for use in the medical field (e.g., for delivery of chemotherapeutics) [4,5], particle size and the particulate fractions of air pollution were beginning to be associated with adverse health effects from a public health perspective [6-8]. Early work in nanotoxicology studied effects of inhaled NPs since epidemiological evidence suggested adverse health effects were associated with poor air quality [6-8]. Studies with isolated ultrafine particulate fractions of air pollution and engineered NPs began to emerge using bronchial cells and macrophages as model cell types [9-11]. Nanotoxicity assays mirrored traditional in vitro assays to understand toxicological impacts of drugs and molecules, with particular emphasis on quantification of cell death and viability, as well as proliferation. 
As one would imagine, there are numerous ways to measure toxicity of a NP, drug or compound in vitro and this is frequently referred to as cytotoxicity testing. Cytotoxicity measurements are typically the first step in assessing safe and biocompatible dose of a drug or material. Cytotoxicity assays are applicable for testing biocompatibility and toxicity of NPs consisting of various compositions and base materials, including dendrimers, metallic, liposomes, micelles, polymeric, carbon-based and others. In monolayer cytotoxicity assays, either primary cells or transformed cell lines are plated into tissue culture plates and acclimated. Depending on the system of interest and intended delivery site, different cell types (derived from liver, lung, skin, kidney, brain heart, etc.) are assessed. The NP is suspended in culture media or appropriate vehicle (phosphate-buffered saline or, in some cases, water) and delivered to the cells at increasing concentrations in order to ascertain dose-dependent toxicological responses. The time of exposure is variable, and it is recommended to obtain data from various time points (e.g., 2, 4, 8, 16, 24 and $48 \mathrm{~h}$, or longer) to gain a temporal understanding of cellular responses to a NP formulation. Quantitative measurements of cellular health are taken using various direct and indirect methods. Common cytotoxicity assays for assessing cell viability include lactate dehydrogenase quantification in cell supernatants and trypan blue staining of exposed cells as a measure of cell membrane permeability, tetrazolium-based (e.g., MTT and WST) assays, which measure mitochondrial activity enzymatically, and esterase cleavage assays such as calcein, and based off of fluorescent signal emission in living cells. Thymidine incorporation (tritium or ${ }^{3} \mathrm{H}$ assay) can be used to measure proliferation. Here, cells are incubated with tritiated thymidine $\left({ }^{3} \mathrm{H}\right.$-Thy). When the cells proliferate, ${ }^{3} \mathrm{H}$-Thy incorporates into new DNA that is synthesized and quantified using a scintillation counter. Other assays are also conducted as a direct measure of cell death. Apoptosis is measured either colorimetrically using ELISA is to quantify caspase activation, or by the appearance of DNA laddering (gel electrophoresis) or fragmentation (TUNEL assay). Apoptosis is also frequently quantified via flow cytometry using markers such as Annexin V. Flow cytometry is a preferred method for toxicological assessments because numerous factors can be assessed at the same time, and an investigator can get multiple pieces of data about the mechanism of cell death from one experiment.

In addition to toxicity profiles, many investigators collect additional data including cellular responses to NP exposures. Proinflammatory cytokine production is often quantified by ELISA, and many NPs have shown their mechanism of action to be driven by reactive oxygen species (ROS) formation [9,11-13], which causes oxidative stress and upregulation of transcription factors associated with inflammation (e.g., NF-kB). The role of ROS in toxicity of NPs has been thoroughly investigated and is reviewed by Abdal Dayem et al [14]. Quantification of ROS upon NP exposure is performed most frequently using dichlorofluorecein diacetate staining [15] and spin trapping method with quantification via electron paramagnetic resonance [16-18].

Since size is a primary factor driving the unique properties displayed by NPs and NPs are often designed to enter cells for intracellular cargo delivery [19,20], it is vital to also understand the molecular toxicity of NPs. One common assay for understanding the molecular toxicity of NPs is the comet assay, which measures DNA strand breaks; quantification of phosphorylated histone H2AX using antibodies and immunofluorescence detection through microscopy or flow cytometry is used to enumerate double-strand breaks [21]. A schematic outlining how NPs interact with cells and cellular outcomes can be found in Figure 1.

More recently, the broader genomic, transcriptomic, metabolic and proteomic profiles of cells exposed to NPs have been investigated and provide a more mechanistic and systematic approach to understanding impacts of NP exposure [22].

\section{In vivo nanotoxicity models}

After acquisition and analyses of in vitro cytoxicity data is complete, the next logical step in determining the safety and efficacy of a NP formulation is to test them using an appropriate animal model. This allows the investigator to gain a better understanding of the behavior of a NP formulation in a complex organism. Parameters such as effective concentration, lethal concentration, and biodistribution in local and distal tissue are measured. Pharmacokinetic parameters are measured or calculated and include - area under the curve (AUC): represented as an integral of the concentration-time curve after a single dose or in steady state; $\mathrm{C}_{\max }$ : the peak plasma concentration of a drug after administration; $T_{\max }$ : time to reach $\mathrm{C}_{\max }$; drug concentration: the amount of drug in a given volume; dose: the amount of drug administered; and bioavailability: the systemically available fraction of a drug. The starting exposure doses that are tested are often extrapolated from in vitro findings, and dose response pilot data are collected to ascertain the timing, frequency and concentration of dosing [23]. Choosing the best animal model that is appropriate for the intended application of the NP formulation can be difficult. However, in general, for engineered NP toxicological assessments, the rodent model is most commonly used before advancing to larger mammalian 


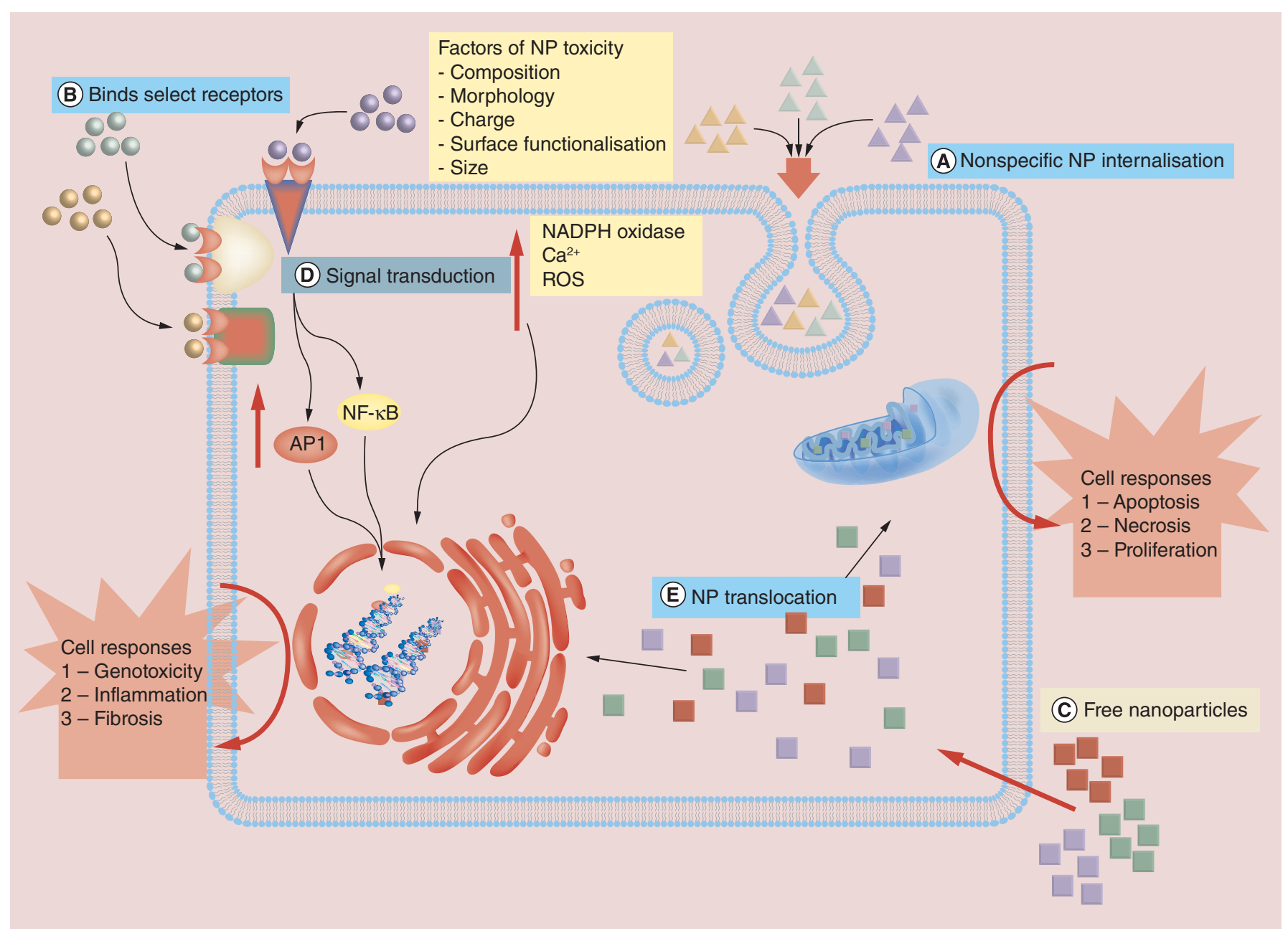

Figure 1. Factors driving cytotoxicity of nanoparticles and cellular responses. Particles are internalized via different mechanisms (A-C) depending on NP properties. The NPs can degrade in endosomes and lysosomes, or interact freely with cellular machinery (D \& E). Cellular responses including effects on transcription and protein expression and genotoxicity which drive cellular responses such as survival, proliferation, apoptosis and necrosis.

NP: Nanoparticle; ROS: Reactive oxygen species.

subjects such as swine, rabbit and nonhuman primates. For an example of an in depth rodent NP exposure experimental design, please refer to Su et al. [24]. Additionally, specific models have been established to investigate other aspects of NP-derived toxicity, including developmental toxicity using zebrafish [25-27] and Drosophila [28,29]. Routes of exposure play an important part in the fate and interactions of NPs in any model. Routes of exposure include inhalation, oral administration, intravenous, subcutaneous, intramuscular, ocular, mucosal and topical. An investigator may also find it necessary to make comparisons among different routes of exposure prior to beginning larger, more depth studies on the formulation.

Common end points collected during animal experiments include survival, histopathology and compartmentalization, biodistribution, metabolites, local and systemic impacts on tissue (liver and kidney functional biomarkers) as well as assessments of various hematological and biochemical parameters.

As stated earlier, the toxicity of NPs is highly dependent on multiple factors beyond size. Composition and surface coating seem to play a major factor with respect to biodistribution after intravenous administration [30-32]. While the broader organismal effects of inorganic and metallic NPs have been studied in vivo at length, liposomes and micelles are generally not considered a toxicological threat since they are comprised of biologically derived molecules. Instead, they are viewed as a biocompatible way to encapsulate poorly soluble molecules, and enhance their biodistribution patterns. However, surfactants, stabilizers and other molecules used to improve solubility, 
including Cremophor, should be investigated alone and as a control set. In fact, Cremophor has been shown to induce adverse effects [33], activate complement cascade [34] and can confound findings.

\section{Toxicity of immunosuppressant nanoformulations}

Given that an overview of common methods for measuring NP toxicity has been provided, the paper will now transition to assessments of immunosuppressant nanoformulation toxicity. Focus will be narrowed to cyclosporine A, rapamycin, and tacrolimus since they are commonly prescribed therapies after transplantation; a very thorough review of immunosuppressants as nanomedicines and their preclinical pharmacologic profiles was also recently published [35].

\section{Cyclosporine A}

Cyclosporine A was first isolated from Tolypocladiuminflattum and selectively targets helper T-cell proliferation while sparing suppressor $T$ cells $[36,37]$. The mechanism of action is well understood via calcineurin inhibition [38]. It is available in two forms: Sandimmune, prepared using water emulsion, and as a microemulsion (Neoral), which has demonstrated improved oral bioavailability [39,40]. Cyclosporine A is given via two routes: oral or intravenous. Oral administration, though preferred, has challenges due to the low bioavailability and high degree of lipophilicity of the drug [41]. Additionally, cyclosporine A has a narrow therapeutic index and dosing is difficult among subjects due to differences in metabolic and absorption efficiency [42]. Furthermore, there are significant side effects associated with cyclosporin A use including hypertension [43] and nephrotoxicity [44]. In whole blood, CsA compartmentalizes to erythrocytes (58\%), plasma (33\%), granulocytes (4\%) and lymphocytes (5\%). In plasma, it preferentially binds to lipoproteins. Higher concentrations are found in lymphatic tissue (spleen, lymph nodes and thymus), compared with blood. It also compartmentalizes to fatty tissues such as liver, pancreas, adrenal glands and adipose tissue [45].

Cyclosporine A is metabolized in the liver through CYP450 mechanisms (specifically by CYP3A4) and excreted via the biliary system [46] as opposed to kidney [47]. Cyclosporine A is cleared at a rate of approximately $0.35 \mathrm{l} / \mathrm{h}$ per $\mathrm{kg}$ and the elimination half-life of the drug varies significantly among patients $(\sim 6-20 \mathrm{~h}$ depending on the patient) [48]. Its cyclic structure resists degradation, and over 30 metabolites have been identified, some of which enhanced the immunosuppressant properties of cyclosporine A while others perpetuate its toxicity [41].

In an effort to enhance solubility of cyclosporine $\mathrm{A}$, a number of agents have been tested in order to increase water solubility of the drug including various nanoformulations. In 2007, Italia et al. [49] used an emulsion/diffusion/evaporation method to prepare poly(lactide-co-glycolide) (PLGA) NPs loaded cyclosporine A and stabilized with didodecylmethylammonium bromide. These particles were $143.3 \pm 8.7 \mathrm{~nm}$ in size by dynamic light scattering and demonstrated $20 \%$ initial drug loading efficiency, with sustained release over a 23 day period. In vitro testing using red blood cell lysis as a marker of biocompatibility revealed that the solubilizer Cremophor alone and Neoral caused significant hemolysis whereas the empty PLGA NPs and PLGA-cyclosporine NPs (at 2 and $10 \mu \mathrm{g} / \mathrm{ml}$ ) did not cause lysis. However, PLGA-cyclosporine NPs at $100 \mu \mathrm{g} / \mathrm{ml}$ displayed increased lysis compared with the lower doses and controls [49]. In situ uptake was greater for PLGA-cyclosporine NPs versus Neoral and cyclosporine A in suspension. Rats treated with PLGA-cyclosporine NPs versus Neoral were found to have increased and sustained plasma concentrations and a delayed $\mathrm{C}_{\max }$ as well as a relative bioavailability of $119.2 \%$, comparatively. At last, neither blank nor PLGA-cyclosporine NPs showed increased in kidney damage as measured by blood urea nitrogen (BUN), creatinine and renal malondialdehyde levels compared with controls, whereas $\mathrm{Ne}$ oral treated rats demonstrated elevated levels of all three markers 31 days post treatment at equivalent cyclosporine concentrations of $15 \mathrm{mg} / \mathrm{kg} /$ day [49]. Ankola et al. [50] used a similar emulsion/diffusion/evaporation process to prepare hydrophilic multiblock copolymer of lactic acid and ethylene glycol containing periodic side-chain carboxyl groups (EL14) NPs for encapsulation of cyclosporine A. In these experiments, cyclosporine A release from EL14 NPs compared with PLGA NPs was found to occur in a time-release fashion over 18 days, were $90 \%$ of the drug was released from EL14 NPs by day 9 compared with $86 \%$ of drug released from PLGA NPs by day 18. In vivo studies conducted with Neoral, PLGA NPs and EL14 NPs containing cyclosporine A. Both PLGA NPs and EL14 NPs were found to have increased bioavailability compared with Neoral, with EL14 demonstrating 5\% greater bioavailability compared with PLGA NPs. After $72 \mathrm{~h}$, the AUC was greatest for EL14 NPs followed by PLGA NPs and Neoral. Though Neoral had the greatest $C_{\max }(1328 \pm 120)$ versus PLGA NPs and EL14 NPs (682 \pm 66 and $1006 \pm 119$, respectively), it had the lowest $\mathrm{T}_{\max }(2 \mathrm{~h})$, compared with EL14 NPs $(12 \mathrm{~h})$ and PLGA NPs $(24 \mathrm{~h})$. After $72 \mathrm{~h}$, the distribution profiles of cyclosporine $A$ were measured after oral administration after one dose at a drug concentration of $15 \mathrm{mg} / \mathrm{kg}$. Tissues harvested from EL14 treated rats were found to have the greatest concentration of drug, with 
the largest amounts found in the liver followed by kidney, spleen, lung, intestine, heart, brain and blood. Interestingly, in comparison with PLGA NPs, the amounts found in the kidney were greater than those in the liver. These findings suggest that cyclosporine A formulations can be prepared in such a manner that is protective in against the digestive enzymes and allow for greater bioavailability when given orally [50]. The immunosuppressant activity of a polymeric micellar formulation (poly[ethylene]-block-poly[€-caprolactone] [PEO-b-PCL] micelles, $\sim 90 \mathrm{~nm}$ ) of cyclosporine A was investigated in vitro using a $\mathrm{T}$ cell and dendritic cell functional assays as well as investigating their function in a mouse model [51]. Activation of dendritic cells was measured by flow cytometry after $72 \mathrm{~h}$ of exposure to either PEO-b-PCL micelle cyclosporine A or Sandimmune. PEO-b-PCL micelle cyclosporine A were found to decrease allostimulatory dendritic cells as well as increase secretion of TGF-B compared with controls. In addition, proliferation of $\mathrm{T}$ cells was found to decrease at a lower concentration as measured by tritium assays. In vivo profiles of immune cell function mirrored the results of the T-cell proliferation and dendritic cell phenotype assays, after mice were injected at a concentration of $20 \mathrm{mg} / \mathrm{kg} /$ day for 3 days, though no histological data were presented. The authors propose that this formulation performs comparable with the Sandimmune formulation and display prolonged circulatory rates [51]. A study by Tang et al. [52] reported the synthesis of cyclosporine A encapsulated within poly(ethylene glycol)-b-poly(d,l-lactide-co-glycolide) (PEG-PLGA) NPs protected by bovine serum albumin. These NPs ( $\sim 90-110 \mathrm{~nm}$, depending on postsynthesis processing) were testing for cyclosporine A release over time in vitro, and approximately $80 \%$ was released by day 2 . In vitro assays investigating T-cell proliferation by tritium counts revealed a dose-dependent decrease in T-cell proliferation when testing doses ranging from 10 to $5000 \mathrm{ng} / \mathrm{ml}$. Proliferation rates were affected in a similar manner compared with free cyclosporine A treated mixed lymphocyte reactions [52]. Similar to the emulsion/diffusion/evaporation described above, another group synthesized pegylated chitosan modified lipid NPs for cyclosporine A encapsulation [53] since chitosan is widely recognized as biologically safe. These liposomal pegylated chitosan NPs were found to be approximately $90 \mathrm{~nm}$ in size with approximately $70 \%$ encapsulation efficiency. Approximately $30 \%$ of cyclosporine A was released within $48 \mathrm{~h}$ in vitro. In vivo studies with rabbits, cyclosporine A plasma concentration was found to be greater after $24 \mathrm{~h} \mathrm{for}$ rabbits treated with pegylated chitosan NPs versus nonpegylated chitosan NPs and cyclosporine alone. Clearance was substantially greater in rabbits treated with cyclosporine A solution after intravenous injection, and AUC was found to be greater 25-times for the pegylated chitosan cyclosporine A NPs compared with cyclosporine in solution. These findings indicate that modification by pegylation may increase circulation time and bioavailability of the drug [53]. Guada and team published several detailed studies investigating lipid NP formulations of cyclosporine A [54-56]. Using hot homogenization and sonication, lipid NPs containing cyclosporine A were synthesized and found to be between 120 and $200 \mathrm{~nm}$; after freeze drying, their size increased to between 163 and $270 \mathrm{~nm}$, and was dependent on stabilizer type [54]. Encapsulation was nearly $100 \%$ and in vitro tests demonstrated that the lipid NPs were not cytotoxic (measured with MTT) but did decrease IL-2 secretion (measured by ELISA) a vital cytokine known to drive T-cell proliferation compared with empty liposomes and Neoral at concentrations of 10 and $25 \mathrm{ng} / \mathrm{ml}$ [54]. The group further investigated the effects of these stable, lyophilizable formulations in a series of in vitro and in vivo tests to gain perspective on the pharmacodynamics and safety of their cyclosporine A NP formulation [55]. In these studies, Caco-2 cells were exposed to increasing concentrations of NPs and analyzed by MTT. Female mice were then exposed with 5 or $15 \mathrm{mg} / \mathrm{kg}$ of drug administered orally through drinking water once a day by gavage. IC50 results in Caco-2 cells revealed that lipid NPs stabilized with tween were less toxic, and chosen as the carrier for the subsequent rodent studies. After 10 days of treatment, mice treated with cyclosporine A NPs demonstrated significantly less lymphocyte numbers compared with unloaded and Neoral treated mice. Additionally, after 5 days of oral treatment, significantly higher and sustained concentrations of cyclosporine A were found in the blood of tween stabilized lipid NPs versus Neoral treated mice $(15 \mathrm{mg} / \mathrm{kg})$. Finally, this study demonstrated that the lipid NP formulations resulted in significantly less renal pathology compared with Neoral treated mice, a finding that can be exploited to extend the therapeutic window of the drug [55]. A comprehensive analysis of the pharmacokinetic behavior of these particles was also performed by this group [56]. Several types of lipid NPs with different stabilizes and fresh or lyophilized were administered as a single oral dose at a concentration of $10 \mathrm{mg} / \mathrm{kg}$ in mice. Those lipids stabilized with Tween were found to have the highest AUC and $\mathrm{C}_{\max }$ compared with Neoral, NPs stabilized with Pluronic, phosphatidylcholine or taurocholate. Those treated with tween stabilized liposomal NPs demonstrative a relative oral bioavailability that was 149\% that of Neoral treated mice [56].

In sum, these expansive studies have demonstrated the feasibility and enhanced properties of NP encapsulated cyclosporine $\mathrm{A}$ and provide proof of principal that there is room to improve upon currently available oral formulations of cyclosporine A. 


\section{Rapamycin}

Rapamycin (sirolimus) is derived from Streptomyces, and functions as an immunosuppressant through inhibition of several cytokine-induced signal transduction pathways (IL-2, -4, -7, -12 and -15) by complexing the FKBP12 intracellular protein and mTOR (mammalian target of rapamycin) protein [57]. Disabling mTOR inhibits downstream synthesis of P70S6K; therefore, the activation of the ribosomal protein S6 does not occur. The inhibition of mTOR also prevents complex formation with Cyclin E, causing inhibition of the T-cell transfer from the G1phase to the S-phase of the cell cycle, effectively suspending T-cell division [58,59]. Additionally, rapamycin inhibits lipopolysaccharide induced proliferation of B cells through a calcium-independent pathway [60,61]. Rapamycin has been packaged into a tablet (Rapamune ${ }^{\circledR}$ ) using NanoCrystal ${ }^{\circledR}$ technology, which involves ball milling to achieve ultrafine particle distributions in the nano range [62], and numerous studies have demonstrated equivalence and bioavailability of Rapamune when compared with liquid oral doses of rapamycin [63-65]. Rapamycin absorption rates when consumed orally are poor (14\%) with a peak blood concentration time $\left(\mathrm{T}_{\max }\right)$ of $1-2 \mathrm{~h}$ [66]. After absorption, much of the drug is partitioned into red blood cells and approximately $40 \%$ will bind to intravascular proteins (albumin) while in circulation [67]. Low oral bioavailability is due to its susceptibility to gastric acid as well as undergoing a first pass elimination by the intestinal P-gp efflux pump and the hepatic CYP450 system (CYP3A4) attributing to its low and variable bioavailability [66]. Additionally, the drug has a lipophilic structure which translates to a large apparent volume of distribution $(1.7 \mathrm{l} / \mathrm{kg})$ and a long half-life $(57-72 \mathrm{~h})$ [66,67]. Biotransformation into numerous metabolites via hydroxylation or demethylation additionally contributes to this lengthened halflife [66]. The parent drug and its metabolites are largely excreted by way of feces $(\sim 98 \%)$ and very little via urine (2\%). Orally delivered rapamycin has exemplified large intra- and interpatient variability and a narrow therapeutic window $(5-15 \mathrm{ng} / \mathrm{ml})[68]$. The toxicity profile of rapamycin administration is dose dependent and has been shown to lead to increased levels of cholesterol, inhibition of bone growth, increased creatinine serum levels as well as decreased glomerular filtration rate, leukopenia, thrombocytopenia, anemia, rash, stomatitis, arthralgia, diarrhea, hypertension and hypokalemia [57].

Bisht $e t$ al. [69] developed N-isopropylacrylamide, methylmethacrylate and acrylic acid (NMA) based polymeric NPs as a carrier for rapamycin and analyzed their pharmacokinetic profile in mice orally at a concentration of $15 \mathrm{mg} / \mathrm{kg}$ compared with Rapamune. NMA NPs were found to be approximately $80 \mathrm{~nm}$ in diameter; rapamycin release was found to be $\mathrm{pH}$ dependent and acidic conditions resulted in $70 \%$ release in $8 \mathrm{~h}$ whereas neutral $\mathrm{pH}(7.4)$ promoted steadier release over time and approached $60 \%$ within $24 \mathrm{~h}$. Blood concentrations of rapamycin were nearly four-times higher compared with Rapamune treated animals after $4 \mathrm{~h}$ and was still detectable at $500 \mathrm{ng} / \mathrm{ml}$ after $24 \mathrm{~h}$. No lung, kidney or liver pathologies were observed after 4 weeks of treatment of empty NMA particles, demonstrating their biocompatibility as a drug carrier. In a xenograft pancreatic cancer model, the NMA rapamycin formulation substantially reduced tumor burden in a fashion that was equivalent to Rapamune suggesting that this polymeric-based delivery system of rapamycin may prolong therapeutic circulation [69].

Nano-based approaches have been taken to encapsulate rapamycin using poly( $\epsilon$-caprolactone)-poly(ethylene glycol)-poly( $\epsilon$-caprolactone) (PCEC) [70]. In these studies, the solubility of PCEC-encapsulated rapamycin (169$180 \mathrm{~nm}$ in size) was increased by 1000-times compared with unencapsulated rapamycin; additionally, the release of PCEC-encapsulated rapamycin was measured by dialysis and was steady over a $48 \mathrm{~h}$ period. Cytotoxicity testing was performed on a large range of doses $(0.05-10 \mathrm{mg} / \mathrm{ml})$ and no toxicity was observed in two different ocular cells, as measured by MTT assay. Rapamycin has also been packaged using poly(D,L-lactic-co-glycolic acid) (PLGA) [71]. Encapsulation efficiency was $81.15 \pm 7.64 \%$ and PLGA was loaded with 500 ng of rapamycin per mg PLGA. The particles were an average size of $280 \mathrm{~nm}$. In these studies, the maturation and viability of bone marrow derived dendritic cells were studied. Cell death was quantified using annexin V staining as an apoptotic marker and secretion of cytokines also assessed. No difference in toxicity was noted for DCs treated with free rapamycin or encapsulated, through the maturation and cytokine secretion of DCs was altered [71,72] and demonstrated potential for further in vivo studies. Another strategy for incorporating rapamycin applied Carbopol 940 at different concentrations to act as a stabilizer for rapamycin loaded PLGA NPs [73]. In this study, the NPs were found to be $200 \mathrm{~nm}$ in size, very stable, with sustained rapamycin release over 30 days that was dependent on the amount of stabilizer. Viability was tested in an endothelial cell culture model by MTT and the particles were shown to have a size dependent reduction in viability [73]. Cho et al. optimized a solid self-microemulsufying drug delivery system (SMEDDS) approach for delivery of rapamycin [74]. After evaluating a panel of stabilizers, the group determined that D- $\alpha$ tocopheryl polyethylene glycol 1000 succinate was most suitable at very acidic $\mathrm{pH}$ (1.7), and final formulation 
(Capryol ${ }^{T M}$ PGMC:glycofurol:vitamin E TPGS, $\sim 108 \mathrm{~nm}$ ) as solid and liquid were used for further bioavailability assessments and compared with raw rapamycin and Rapamune. Blood concentrations of rapamycin after exposure to the SMEDDS were comparable with Rapamune exposed rats. The Capryol ${ }^{T M}$ PGMC:glycofurol:vitamin E TPGS liquid SMEDD formulation was shown to have higher AUC and double the $\mathrm{C}_{\max }$ compared with Rapamune oral formulation and more than 12-times greater $\mathrm{C}_{\max }$ compared with raw rapamycin powder [74]; further studies from this same group also investigated supersaturatable formulations and screening with similar results [75,76].

While a good deal of research has focused on strategies to make rapamycin more bioavailable in oral formulation, other researchers have set out to target rapamycin NP formulations to site-directed locations in an effort to attenuate endothelial cell-mediated inflammation [77]. In this study, micelles were loaded with rapamycin and decorated with cyclic arginine-glycine-aspartate moieties to facilitate targeting to integrin $\alpha \mathrm{V} \beta 3$ on endothelial cells. Untargeted NPs were approximately $10 \mathrm{~nm}$ while those with cyclic arginine-glycine-aspartate were about $15 \mathrm{~nm}$. Release of rapamycin was found to be $\mathrm{pH}$ dependent and peaked at $\mathrm{pH}$ 7. In vitro tests by MTT showed no cytotoxicity of empty micelles, loaded with rapamycin (targeted or untargeted), or free rapamycin up to concentrations of $3 \mu \mathrm{g} / \mathrm{ml}$ after 6 h. Additionally, in vitro targeting demonstrated, and inflammatory markers (IL-6 and IL-8) were decreased; these results were confirmed in a C57 mouse model [77]. This same group recently provided evidence that this targeted formulation could be used as an additive and pretreatment regimen to prevent chronic rejection in vivo [78].

Collectively, multiple novel approaches have validated the need for further development efficacious strategies for encapsulation of hydrophobic immunosuppressant drugs in nanoformulations.

\section{Tacrolimus}

Tacrolimus remains the mainstay in the majority of transplantation drug regimens. The mechanism of action of tacrolimus as an immunosuppressant has been extensively studied [79] and mainly driven by calcineurin phosphatase inactivation [80] via binding to the intracellular protein FKBP12 [81]. This inactivation prevents the transport of nuclear factor of activated T cells (NF-AT), which is required for T-cell activation and expression of several cytokines, including IL-2. Decreased IL-2 reduces the number of circulating activated alloreactive T cells, thereby preserving the graft [80]. However, it displays a narrow therapeutic range, with pharmacokinetic patterns resulting in blood level fluctuation (diurnal peaks and troughs) after oral administration. Absorption of oral tacrolimus AUC can vary significantly (average of 25\% and ranging from 5 to $67 \%$ [82]), with inconsistencies in intestinal absorption (fasting vs high fat meals) or skipped doses due to noncompliance may lead to recurrent under immunosuppression with increased risk of acute or chronic rejection episodes. Distribution after absorption appears to vary drastically with representative sites being erythrocytes, lungs, liver, kidneys, pancreas, heart and spleen. Tacrolimus is metabolized in the liver via the CYP3A4 and CYP3A5 isoenzymes in addition to the P-gp efflux pump [81] in the intestinal wall to eight possible metabolites, with the 31-demethyl tacrolimus being the most common which has similar efficacy as tacrolimus [83]. The half-life of tacrolimus is extremely variable from patient to patient $(2.1-36 \mathrm{~h})$ with a time to peak onset of $0.5-6 \mathrm{~h}$. Tacrolimus is primarily excreted at an average rate of $30 \mathrm{ml} / \mathrm{min} / \mathrm{kg}$ in the feces $(93 \%)$ and $<1 \%$ is found to be excreted via the kidney in the active form of the drug [84]. Attempts to restore trough levels to therapeutic range may result in over immunosuppression with toxic risks such as nephrotoxicity, malignancy or opportunistic infection [85] - among many others [82]. Consistent and reliable maintenance of patients within a therapeutic range that is not too high or too low is thus extremely challenging. Additionally, systemic tacrolimus levels do not proportionally correlate with local graft concentrations, with significant interpatient and intrapatient variability in drug exposure at comparable doses $[23,86,87]$.

Like rapamycin and cyclosporine $\mathrm{A}$, tacrolimus has been the target of many nanomedicine strategies in order to increase its bioavailability and reduce nephrotoxicity [35]. An early study investigating tacrolimus loaded PLGA NPs entrapped into pH-sensitive microspheres (NPMS; $36 \mu \mathrm{m}$ microspheres containing $243 \mathrm{~nm}$ NPs) demonstrated increased drug release at $\mathrm{pH} 7.4$ versus $\mathrm{pH}$ 4, and bioavailability was also reduced in the NPMS formulation compared with oral solutions; however, this result was anticipated and the intent of the design for treatment of colitis [24]. Tacrolimus has been encapsulated into biodegradable poly(ethylene glycol)-poly(D,L lactide) (MPEGPLA) block copolymers using a double emulsion-solvent evaporation yielding approximately $90 \mathrm{~nm}$ [25]. Empty NPs were found to have very little toxicity in a kidney cytoxicity model, even up to $1000 \mu \mathrm{g} / \mathrm{ml}$ as measured by MTT. Over time, tacrolimus concentration in plasma of rats exposed after gastric perfusion was increased compared with capsulated tacrolimus. More importantly, in a liver transplant model, rats were shown to live significantly longer ( $80 \%$ survived 20 days compared with only $40 \%$ of the tacrolimus capsule treated rats) with significantly less alanine aminotransferase concentrations in the NP treated group [25]. 
Preparation of liposomal formulations of tacrolimus has been prepared in the absence of organic solvents [26]. This formulation was tested for safety in human subjects after a single intravenous dose of $0.025 \mathrm{mg} / \mathrm{kg}$ to compare the liposomal formulation pharmacokinetics and a HCO-60 (polyoxyl 60 hydrogenated castor oil (HCO-60) and ethanol; Prograf) based formulation currently on the market. No differences were found in that study, though the number of subjects was only six per group. In a mouse study, circulating lymphocyte numbers were not changed, and repeated intravenous doses at a concentration of $2.5 \mathrm{mg} / \mathrm{kg}$ did not cause death, weight loss, histopathologic or hematologic changes. The same results were found in rats, up to a dose of $1.25 \mathrm{mg} / \mathrm{kg}$ over 14 days of repeated intravenous exposure [26]. The findings from this study appeared to provide initial evidence that liposomal tacrolimus formulation could benefit transplant patients, though further testing must be completed. Tacrolimus loaded into albumin NPs were constructed using an emulsification-dispersion method, resulting in NPs approximately $190 \mathrm{~nm}$ in size [27]. A study was conducted in rats using a single intravenous injection at a concentration of $10 \mathrm{mg} / \mathrm{kg}$. In comparison with Prograf, this formulation demonstrated increased AUC $(151.5 \pm 46.69$ vs $84.4 \pm 10.98 \mathrm{~min}$ $\mathrm{mg} / \mathrm{l}$ for the NP vs Prograf treated animals, respectively) as well as increased mean residence time and decreased clearance. In blood samples, the NP-treated rats demonstrated higher tacrolimus concentrations over a $24 \mathrm{~h}$ period. Significantly, lower kidney to blood ratios were also notes for NP treated rats at $0.5,10$ and $24 \mathrm{~h}$ post injection; importantly, markers for kidney damage were significantly less compared with Prograf, but still higher than control groups after 14 days of treatment with $0.8 \mathrm{mg} / \mathrm{kg} /$ day, indicating that while improved, nephrotoxicity is still a side effect risk [27]. Other groups have also demonstrated the feasibility of tacrolimus-loaded albumin NPs [28] and their applicability for arthritis treatment in mice. Most recently, Zhang et al. [29] published results from an albumin-based tacrolimus NP that demonstrated prolonged circulation and decreased renal toxicity.

\section{Challenges in proper assessment of NP toxicity}

While there are numerous ways one can determine the overall toxicity of a NP, it is very important to utilize the proper controls. It is important to note that NP variables such as charge, composition, surface functionalization, size and shape can alter cellular interactions based on cell type, thereby resulting in different toxic profiles and interferences. Many studies have demonstrated that NPs can cause false negative results by binding proteins that are being measured [30,31], inhibiting enzymatic reactions [32] or absorbing fluorescent signals [33,34]. When gathering in vitro data on the toxic potential of a compound or NP, it is important to use more than one assay as well as properly characterize the absorption and emission spectrum of the drug to ensure minimal interference with colorimetric and fluorescence assays.

NPs have been shown to enter cells via a variety of mechanisms including receptor mediated endocytosis, an angle that has been exploited for site specific delivery and reviewed by Xu et al [88]. Nonspecific uptake of NPs has been demonstrated in macrophage cultures [89], with charge and size emerging as driving factors. Indeed, formation of a protein corona on the surface of NPs has been shown to cause differences in toxicity and can alter NP internalization pathways leading to engulphment through the endosomal/lyosomal pathway [90-93]. Adhesion of albumin and other serum proteins found in cell culture media can modulate surface properties of NPs leading to changes in aggregation and charge, as evidenced by Choi et al., who demonstrated that toxicological profiles including CYP protein expression in hepatic cells were altered in the presence of a protein corona that formed on gold NPs [94]. Accordingly, it is advisable to perform physicochemical characterization assays of NPs in the vehicle that the NPs will be delivered to cells in. For example, studies performed with quantum dots in RPMI with fetal bovine serum, RPMI alone and serum free media with supplements demonstrated that surface $\zeta$ potential and size were significantly changed, and dependent on the capping molecules [95]. Aggregation of positively charged quantum dots was noted and pathway of cellular internalization could not be identified due to interference of the spectrometric signal and the NPs.

Dos Santos et al. tested various cell types including astrocytes, macrophages, epithelial cells and endothelial cells and determined that size of NP and cell type both affect uptake kinetics [96]. Similar to cytotoxicity assays, there can be considerable interferences with genotoxicity and mutagenic assays [97,98]. George et al. assessed assay interference for chromosome aberration, micronucleus formation, and comet assay after Chinese hamster ovary cells were exposed to gold NPs for $20 \mathrm{~h}$. Interference was noted for the comet assay but not the other tests [98]. As noted above, a common assay to determine oxidative stress in cells exposed to NPs is the dichlorofluorescein diacetate fluorescent probe. Pal et al. performed a series of well-controlled experiment and noted several factors that can lead to aberrant interpretation of results including NP concentration, emission spectra absorption by NP and 
auto oxidation of the dye [99]. This again underscores the necessity of conducting multiple cytotoxic and genotoxic assays with appropriate controls before drawing conclusions regarding NP toxicity and biocompatibility.

Most of the experiments described in the immunosuppressant nanoformulation discussion above provided detailed information on the synthesis of NPs. They also provided methods for NP characterization and standard methods for testing drug release in vitro. Most also provided data from toxicity testing of empty NPs. It is important for researchers to test all variables that could affect toxicity including the carrier materials. Proper controls, including standard-of-care comparisons should be included. While the data summarized above did not provide an exhaustive presentation of all efforts to encapsulate immunosuppressants into nanoformulations, several key studies have been described for the major immunosuppressant therapies given to transplant patients. Further experiments are needed particularly with respect to bioavailability of tacrolimus nanoformulations given through an oral route. More complex studies detailing the impacts of these formulations on immune responses after chronic exposures as well more evaluations on side effects such as hypertension and gastrointestinal (GI) inflammation need to be conducted.

\section{Future directions for NP toxicity assessments}

This next section focuses on next generation models and other approaches for assessing toxicity and biocompatibility of NP formulations. One approach that has rapidly evolved is the generation of more complex and relevant tissue culture models that integrate multiple components of an organ system. For example, advanced coculture systems have been developed in order to gain more insight into the complex interactions between NPs and different cell types and tissue. There are well-developed 3D coculture and organoid models for testing NP toxicity in lung [100-103], brain [104], kidney [105] and liver [105,106]. While numerous examples of coculture models for nanotoxicity exist in the literature (reviewed in [107]), of particular relevance to investigate toxicity of nanomedicines for applications in transplant biology are models for assessing GI interactions, absorption, integrity and toxicity. A sophisticated 3D model was developed to simulate the GI tract and interactions with NPs [108]. Here, gold, silver and titanium NPs were applied to cocultures that were noninflamed, or stimulated with IL-1B to induce an inflammatory environment. Toxicity was found to increase during inflamed conditions, as measured by IL-8 production [108]. Conversely, a comparison of Caco-2 monolayers, coculture of Caco-2 cells with mucus secreting cells, and a triculture which also added $\mathrm{M}$ cells demonstrated variance in translocation rates of charged NPs in the coculture with mucosal cells; however, this difference was not apparent in the triculture model with all three cells types [109]. Chia et al. [110] developed a 3D organoid model of the colon and compared the toxicity of zinc NPs against monolayer models and found that the more simplistic monolayer exposures may overestimate toxic potential of NPs. Another example of an appropriate GI model for 3D in vitro assessment of NP toxicity was put forth by Ude et al., who studied toxicity of copper NPs in mucus secreting and microfold Caco-2 cocultures. In this study, the cocultures were found to be less sensitive to NP exposure (as measured by IL-8 secretion and barrier integrity) compared with NP monolayer exposures [111]; this important finding suggests that while in vitro tests can provide valuable data about the potential toxicity of a NP formulation, there is benefit in creating more physiologically relevant and sophisticated models for in vitro testing and also confirmed results in animals that are challenged with oral formulations to determine stability. With increasing interest in developing oral formulations of immunosuppressant drugs, a relevant coculture 3D model of GI interactions could serve as a valuable tool, particularly for individuals who might suffer from GI side effects related to transplant.

As with any experimental field, there are hurdles in the field of toxicology to be overcome, and no one assay can provide all information needed to make a definitive decision regarding the safety of a compound. Collective cytotoxicity and animal model data are analyzed and risk assessments are performed to determine the safety of a NP or compound.

\section{Future perspective}

The future of transplant medicine is bright. Many of the strategies for encapsulating hydrophobic drugs that have shown promise in the field of oncology and cancer therapeutics are applicable to the transplant community. Toxicological profile concerns for commonly used immunosuppressants can be addressed with proper encapsulation and NP stabilization strategies, allowing for increased therapeutic yields to reach its target. Surface modifications to creatively decorate the surfaces of NPs will enable increased delivery while avoiding nephrotoxicity and hepatotoxicity of common immunosuppressants. Advancements in next generation organ on a chip in vitro models, 
computational toxicology and pharmacologic modeling can also be used to predict biocompatibility of newly designed small molecules and engineered NP formulations for transplant medicine applications.

\section{Executive summary}

- The maturation of nanotoxicology over the past three decades has provided many models, in vitro and in vivo, as well as countless assays to assess the safety, toxicity and biocompatibility of nanoparticle (NP)-based therapeutics.

- It is important to consider numerous parameters when designing nanoformulations, including particle composition, charge, size, surface functionalization, stability (aggregation), morphology and intended target.

- Physico-chemical parameters all have different, yet important, impacts on local and systemic toxicity profiles.

- Route of NP administration directly drives fate and biological responses to exposures.

- Absorption of NPs through ingestion and dermal application of NPs as well as mucosal contact, inhalation, and intravenous injection are potential pathways for NPs to enter the body.

- Systemic properties can be both exploited for site-directed delivery but also vulnerable to unwanted effects such as inflammation and accumulation, which can be dependent on the metabolism of the NP.

- Cyclosporine, rapamycin and tacrolimus have been successfully incorporated into various nanoencapsulation systems and have demonstrated reduced toxicity compared with traditional forms of this immunosuppressants.

- New and more complex in vitro toxicity models as well as advancements in predictive toxicity will aid in future risk assessments for immunosuppressant safety and efficacy.

- Ultimately, with thoughtful and careful design, nanoformulations can be created that achieve safer and more efficacious doses, fewer side effects, enhanced bioavailability and therefore more predictable therapeutic responses, and targeted delivery.

Author contributions

A Nagy created the outline and structure of the manuscript as well as wrote sections on nanotoxicity models and nanoformulations of immunosuppressants. N Robbins edited the document and assisted with writing toxicity profile of immunosuppressant pharmacokinetics.

\section{Acknowledgments}

The authors thank J Janjic, V Gorantla and $\vee$ Sylvia for their critical review of this manuscript. We also thank T Yuan and AM DiGeorge Foushee for their assistance generating art for this manuscript.

\section{Disclaimer}

The views expressed are those of the authors and do not reflect the official views or policy of the department of defense or its components. The views of the manufacturers of the pharmaceutics presented in this document are not necessarily the official views of, or endorsed by, the US Government, the Department of Defense, or the Department of the Air Force. No federal endorsement of the pharmaceutics listed is intended.

Financial \& competing interests disclosure

The authors have no relevant affiliations or financial involvement with any organization or entity with a financial interest in or financial conflict with the subject matter or materials discussed in the manuscript. This includes employment, consultancies, honoraria, stock ownership or options, expert testimony, grants or patents received or pending, or royalties.

No writing assistance was utilized in the production of this manuscript

\section{Open access}

This work is licensed under the Attribution-NonCommercial-NoDerivatives 4.0 Unported License. To view a copy of this license, visit http://creativecommons.org/licenses/by-nc-nd/4.0/

\section{References}

Papers of special note have been highlighted as: $\bullet$ of interest $\bullet \bullet$ of considerable interest

1. Auffan M, Rose J, Bottero JY, Lowry GV, Jolivet JP, Wiesner MR. Towards a definition of inorganic nanoparticles from an environmental, health and safety perspective. Nat. Nanotechnol. 4(10), 634-641 (2009).

2. Cleared nanoparticles for medical use. https://nanohybrids.net/pages/cleared-nanoparticles-for-medical-use

3. Bobo D, Robinson KJ, Islam J, Thurecht KJ, Corrie SR. Nanoparticle-based medicines: a review of FDA-approved materials and clinical trials to date. Pharm. Res. 33(10), 2373-2387 (2016). 
4. Hosoda J, Unezaki S, Maruyama K, Tsuchiya S, Iwatsuru M. Antitumor activity of doxorubicin encapsulated in poly(ethylene glycol)-coated liposomes. Bio. Pharm. Bull. 18(9), 1234-1237 (1995).

5. Storm G, Roerdink FH, Steerenberg PA, De Jong WH, Crommelin DJ. Influence of lipid composition on the antitumor activity exerted by doxorubicin-containing liposomes in a rat solid tumor model. Cancer Res. 47(13), 3366-3372 (1987).

6. Dockery DW, Pope CA 3rd, Xu X et al. An association between air pollution and mortality in six US cities. N. Engl. J. Med. 329(24), 1753 (1993).

7. Pope CA 3rd. Mortality effects of longer term exposures to fine particulate air pollution: review of recent epidemiological evidence. Inhal. Res. 19(Suppl. 1), 33-38 (2007).

8. Pope CA 3rd, Burnett RT, Thun MJ et al. Lung cancer, cardiopulmonary mortality, and long-term exposure to fine particulate air pollution. JAMA 287(9), 1132 (2002).

9. Boland S, Hussain S, Baeza-Squiban A. Carbon black and titanium dioxide nanoparticles induce distinct molecular mechanisms of toxicity. Wiley Interdiscipl. Rev. Nanomed. Nanotechnol. 6(6), 641-652 (2014).

10. Leclerc L, Rima W, Boudard D et al. Size of submicrometric and nanometric particles affect cellular uptake and biological activity of macrophages in vitro. Inhal. Toxicol. 24(9), 580-588 (2012).

11. Lu S, Zhang W, Zhang R et al. Comparison of cellular toxicity caused by ambient ultrafine particles and engineered metal oxide nanoparticles. Part. Fibre Toxicol. 12, 5 (2015).

12. Han D-W, Hong SC, Lee JH et al. Subtle cytotoxicity and genotoxicity differences in superparamagnetic iron oxide nanoparticles coated with various functional groups. Int. J. Nanomed. 6, 3219-3231 (2011).

13. Inkielewicz-Stepniak I, Niska K, Pyszka K, Tukaj C, Wozniak M, Radomski M. Titanium dioxide nanoparticles enhance production of superoxide anion and alter the antioxidant system in human osteoblast cells. Int. J. Nanomed.10, 1095-1107 (2015).

14. Abdal Dayem A, Hossain MK, Lee SB et al. The role of reactive oxygen species (ROS) in the biological activities of metallic nanoparticles. Int. J. Mol. Sci. 18(1), 1-21 (2017).

15. He SJ, Cao J, Li YS et al. CdSe/ZnS quantum dots induce photodynamic effects and cytotoxicity in pancreatic cancer cells. World J. Gastroenterol. 22(21), 5012-5022 (2016).

16. Peebles BC, Dutta PK, Waldman WJ et al. Physicochemical and toxicological properties of commercial carbon blacks modified by reaction with ozone. Environ. Sci. Technol. 45(24), 10668-10675 (2011).

17. Villamena FA, Zweier JL. Detection of reactive oxygen and nitrogen species by EPR spin trapping. Antioxid. Redox Signal. 6(3), 619-629 (2004).

18. Angele-Martinez C, Nguyen KV, Ameer FS, Anker JN, Brumaghim JL. Reactive oxygen species generation by copper(II) oxide nanoparticles determined by DNA damage assays and EPR spectroscopy. Nanotoxicology 11(2), 278-288 (2017)

19. Maity AR, Stepensky D. Efficient subcellular targeting to the cell nucleus of quantum dots densely decorated with a nuclear localization sequence peptide. ACS Appl. Mater. Interfaces 8(3), 2001-2009 (2016).

20. Maity AR, Stepensky D. Nuclear and perinuclear targeting efficiency of quantum dots depends on density of peptidic targeting residues on their surface. J. Control. Rel. 257, 32-39 (2017).

21. Wan R, Mo Y, Tong R, Gao M, Zhang Q. Determination of phosphorylated histone H2AX in nanoparticle-induced genotoxic studies. Methods Mol. Biol. 1894, 145-159 (2019).

22. Frohlich E. Role of omics techniques in the toxicity testing of nanoparticles. J. Nanobiotechnol. 15(1), 84 (2017).

23. Sellares J, De Freitas DG, Mengel M et al. Understanding the causes of kidney transplant failure: the dominant role of antibody-mediated rejection and nonadherence. Am. J. Transplant. 12(2), 388-399 (2012).

24. Lamprecht A, Yamamoto H, Takeuchi H, Kawashima Y. A pH-sensitive microsphere system for the colon delivery of tacrolimus containing nanoparticles. J. Control. Rel. 104(2), 337-346 (2005).

25. $\mathrm{Xu}$ W, Ling $\mathrm{P}$, Zhang $\mathrm{T}$. Toward immunosuppressive effects on liver transplantation in rat model: tacrolimus loaded poly(ethylene glycol)-poly(D,L-lactide) nanoparticle with longer survival time. Int. J. Pharm. 460(1-2), 173-180 (2014).

- Provides evidence for a nanomedicine formulation that improves survival and reduced graft rejection rates.

26. Ali SM, Ahmad A, Sheikh S et al. Polyoxyl 60 hydrogenated castor oil free nanosomal formulation of immunosuppressant tacrolimus: pharmacokinetics, safety, and tolerability in rodents and humans. Int. Immunopharmacol. 10(3), 325-330 (2010).

27. Zhao L, Zhou Y, Gao Y et al. Bovine serum albumin nanoparticles for delivery of tacrolimus to reduce its kidney uptake and functional nephrotoxicity. Int. J. Pharm. 483(1-2), 180-187 (2015).

28. Thao Le Q, Byeon HJ, Lee $\mathrm{C}$ et al. Pharmaceutical potential of tacrolimus-loaded albumin nanoparticles having targetability to rheumatoid arthritis tissues. Int. J. Pharm. 497(1-2), 268-276 (2016).

29. Zhang Y, Pan J, Li H et al. Albumin based nanomedicine for enhancing tacrolimus safety and lymphatic targeting efficiency. J. Biomed. Nanotechnol. 15(6), 1313-1324 (2019).

30. Almutary A, Sanderson BJ. The MTT and crystal violet assays: potential confounders in nanoparticle toxicity testing. Int. J. Toxicol. 35(4), 454-462 (2016). 
31. Holder AL, Goth-Goldstein R, Lucas D, Koshland CP. Particle-induced artifacts in the MTT and LDH viability assays. Chem. Res. Toxicol. 25(9), 1885-1892 (2012).

32. Wright PC, Qin H, Choi MM, Chiu NH, Jia Z. Carbon nanodots interference with lactate dehydrogenase assay in human monocyte THP-1 cells. SpringerPlus 3, 615 (2014).

33. Kroll A, Pillukat MH, Hahn D, Schnekenburger J. Interference of engineered nanoparticles with in vitro toxicity assays. Arch. Toxicol. 86(7), 1123-1136 (2012).

34. Pem B, Gonzalez-Mancebo D, Moros $\mathrm{M}$ et al. Biocompatibility assessment of up-and down-converting nanoparticles: implications of interferences with in vitro assays. Methods Appl. Fluoresc. 7(1), 014001 (2018).

35. Al-Lawati H, Aliabadi HM, Makhmalzadeh BS, Lavasanifar A. Nanomedicine for immunosuppressive therapy: achievements in pre-clinical and clinical research. Expert Opin. Drug Deliv. 15(4), 397-418 (2018).

- In depth review of immunosuppressant nanomedicine formulations.

36. Italia JL, Bhardwaj V, Kumar MN. Disease, destination, dose and delivery aspects of ciclosporin: the state of the art. Drug Discov. Today 11(17-18), 846-854 (2006).

37. Liu H, Wang Y, Li S. Advanced delivery of ciclosporin A: present state and perspective. Expert Opin. Drug Deliv. 4(4), 349-358 (2007).

38. Guada M, Beloqui A, Kumar MN, Preat V, Dios-Vieitez Mdel C, Blanco-Prieto MJ. Reformulating cyclosporine A (CsA): more than just a life cycle management strategy. J. Control. Rel. 225, 269-282 (2016).

39. Coukell AJ, Plosker GL. Cyclosporin microemulsion (Neoral). A pharmacoeconomic review of its use compared with standard cyclosporin in renal and hepatic transplantation. Pharmacoeconomics 14(6), 691-708 (1998).

40. Noble S, Markham A. Cyclosporin. A review of the pharmacokinetic properties, clinical efficacy and tolerability of a microemulsion-based formulation (Neoral). Drugs 50(5), 924-941 (1995).

41. Christians U, Sewing KF. Cyclosporin metabolism in transplant patients. Pharmacol. Ther. 57(2-3), 291-345 (1993).

42. Kahan BD. Therapeutic drug monitoring of cyclosporine: 20 years of progress. Transplant. Proc. 36(Suppl. 2), 378S-391S (2004).

43. Textor SC, Canzanello VJ, Taler SJ et al. Cyclosporine-induced hypertension after transplantation. Mayo Clinic Proc. 69(12), 1182-1193 (1994).

44. Ader JL, Rostaing L. Cyclosporin nephrotoxicity: pathophysiology and comparison with FK-506. Curr. Opin. Nephrol. Hypertens. 7(5), 539-545 (1998).

45. Lindholm A. Factors influencing the pharmacokinetics of cyclosporine in mank. Ther. Drug Monitor. 13(6), $465-477$ (1991).

46. Galan AI, Fernandez E, Moran D, Munoz ME, Jimenez R. Cyclosporine A hepatotoxicity: effect of prolonged treatment with cyclosporine on biliary lipid secretion in the rat. Clin. Exp. Pharmacol. Physiol. 22(4), 260-265 (1995).

47. Bleck JS, Schlitt HJ, Christians U et al. Urinary excretion of ciclosporin and 17 of its metabolites in renal allograft recipients. Pharmacology 39(3), 160-164 (1989).

48. Freeman DJ. Pharmacology and pharmacokinetics of cyclosporine. Clin. Biochem. 24(1), 9-14 (1991).

49. Italia JL, Bhatt DK, Bhardwaj V, Tikoo K, Kumar MN. PLGA nanoparticles for oral delivery of cyclosporine: nephrotoxicity and pharmacokinetic studies in comparison to Sandimmune Neoral. J. Control. Rel. 119(2), 197-206 (2007).

50. Ankola DD, Battisti A, Solaro R, Kumar MNVR. Nanoparticles made of multi-block copolymer of lactic acid and ethylene glycol containing periodic side-chain carboxyl groups for oral delivery of cyclosporine A. J. R.Soc. Interface. 7(Suppl. 4), S475-S481 (2010).

51. Hamdy S, Haddadi A, Shayeganpour A, Alshamsan A, Montazeri Aliabadi H, Lavasanifar A. The immunosuppressive activity of polymeric micellar formulation of cyclosporine A: in vitro and in vivo studies. AAPS J. 13(2), 159-168 (2011).

52. Tang L, Azzi J, Kwon M et al. Immunosuppressive activity of size-controlled PEG-PLGA nanoparticles containing encapsulated cyclosporine A. J. Transplant. 2012, 896141 (2012).

53. Zhang L, Zhao ZL, Wei XH, Liu JH. Preparation and in vitro and in vivo characterization of cyclosporin A-loaded, PEGylated chitosan-modified, lipid-based nanoparticles. Int. J. Nanomedicine 8, 601-610 (2013).

54. Guada M, Sebastian V, Irusta S, Feijoo E, Dios-Vieitez Mdel C, Blanco-Prieto MJ. Lipid nanoparticles for cyclosporine A administration: development, characterization, and in vitro evaluation of their immunosuppression activity. Int. J. Nanomed.10, 6541-6553 (2015).

55. Guada M, Lana H, Gil AG, Dios-Vieitez Mdel C, Blanco-Prieto MJ. Cyclosporine A lipid nanoparticles for oral administration: pharmacodynamics and safety evaluation. Eur. J. Pharm. Biopharm. 101, 112-118 (2016).

- Descriptive study of preparation, characterization and in vitro analyses of novel nanoformulation of cyclosporine $A$.

56. Guada M, Lasa-Saracibar B, Lana H, Dios-Vieitez Mdel C, Blanco-Prieto MJ. Lipid nanoparticles enhance the absorption of cyclosporine A through the gastrointestinal barrier: in vitro and in vivo studies. Int. J. Pharm. 500(1-2), 154-161 (2016).

- Follow on in vivo assesment of a novel cyclosporine A formulation; detailed discussion on mechanism of action and function.

57. Sehgal SN. Sirolimus: its discovery, biological properties, and mechanism of action. Transplant. Proc. 35(Suppl. 3), 7S-14S (2003).

58. Sehgal SN. Rapamune (RAPA, rapamycin, sirolimus): mechanism of action immunosuppressive effect results from blockade of signal transduction and inhibition of cell cycle progression. Clin. Biochem. 31(5), 335-340 (1998). 
59. Siekierka JJ. Probing T-cell signal transduction pathways with the immunosuppressive drugs, FK-506 and rapamycin. Immunol. Res. 13(2-3), 110-116 (1994).

60. Kay JE, Kromwel L, Doe SE, Denyer M. Inhibition of T and B lymphocyte proliferation by rapamycin. Immunology 72(4), 544-549 (1991).

61. Wicker LS, Boltz RC Jr, Matt V, Nichols EA, Peterson LB, Sigal NH. Suppression of B cell activation by cyclosporin A, FK506 and rapamycin. Eur. J. Immunol. 20(10), 2277-2283 (1990).

62. Junghanns J-UaH, Müller RH. Nanocrystal technology, drug delivery and clinical applications. Int. J. Nanomed. 3(3), 295-309 (2008).

63. Shen LJ, Wu FL. Nanomedicines in renal transplant rejection-focus on sirolimus. Int. J. Nanomed. 2(1), 25-32 (2007).

64. Mathew TH, Van Buren C, Kahan BD, Butt K, Hariharan S, Zimmerman JJ. A comparative study of sirolimus tablet versus oral solution for prophylaxis of acute renal allograft rejection. J. Clin. Pharmacol. 46(1), 76-87 (2006).

65. Kelly PA, Napoli K, Kahan BD. Conversion from liquid to solid rapamycin formulations in stable renal allograft transplant recipients. Biopharm. Drug Disposition 20(5), 249-253 (1999).

66. Haeri A, Osouli M, Bayat F, Alavi S, Dadashzadeh S. Nanomedicine approaches for sirolimus delivery: a review of pharmaceutical properties and preclinical studies. Artif. Cell. Nanomed. Biotechnol. 46(Suppl.1), 1-14 (2018).

67. Macdonald A, Scarola J, Burke JT, Zimmerman JJ. Clinical pharmacokinetics and therapeutic drug monitoring of sirolimus. Clin. Ther. 22(Suppl. B), B101-B121 (2000).

68. Shihab F, Christians U, Smith L, Wellen JR, Kaplan B. Focus on mTOR inhibitors and tacrolimus in renal transplantation: pharmacokinetics, exposure-response relationships, and clinical outcomes. Transpl. Immunol. 31(1), 22-32 (2014).

69. Bisht S, Feldmann G, Koorstra JB et al. In vivo characterization of a polymeric nanoparticle platform with potential oral drug delivery capabilities. Mol. Cancer Ther. 7(12), 3878-3888 (2008).

70. Zhang Z, Xu L, Chen H, Li X. Rapamycin-loaded poly(epsilon-caprolactone)-poly(ethylene glycol)-poly(epsilon-caprolactone) nanoparticles: preparation, characterization and potential application in corneal transplantation. J. Pharm. Pharmacol. 66(4), 557-563 (2014).

71. Haddadi A, Elamanchili P, Lavasanifar A, Das S, Shapiro J, Samuel J. Delivery of rapamycin by PLGA nanoparticles enhances its suppressive activity on dendritic cells. J. Biomed. Mater. Res. Part A 84(4), 885-898 (2008).

72. Das S, Haddadi A, Veniamin S, Samuel J. Delivery of rapamycin-loaded nanoparticle down regulates ICAM-1 expression and maintains an immunosuppressive profile in human CD34+ progenitor-derived dendritic cells. J. Biomed. Mater. Res. Part A 85(4), $983-992$ (2008).

73. Zou W, Cao G, Xi Y, Zhang N. New approach for local delivery of rapamycin by bioadhesive PLGA-carbopol nanoparticles. Drug Deliv. 16(1), 15-23 (2009).

74. Cho W, Kim MS, Kim JS et al. Optimized formulation of solid self-microemulsifying sirolimus delivery systems. Int. J. Nanomedicine 8, 1673-1682 (2013).

75. Kim MS, Kim JS, Cho W et al. Supersaturatable formulations for the enhanced oral absorption of sirolimus. Int. J. Pharm. 445(1-2), 108-116 (2013).

76. Kim M-S, Kim J-S, Cho WK, Hwang S-J. Enhanced solubility and oral absorption of sirolimus using D- $\alpha$-tocopheryl polyethylene glycol succinate micelles. Artif. Cell. Nanomed. Biotechnol. 41(2), 85-91 (2013).

77. Nadig SN, Dixit SK, Levey N et al. Immunosuppressive nano-therapeutic micelles downregulate endothelial cell inflammation and immunogenicity. RSC Adv. 5(54), 43552-43562 (2015).

78. Zhu P, Atkinson C, Dixit $S$ et al. Organ preservation with targeted rapamycin nanoparticles: a pretreatment strategy preventing chronic rejection in vivo. RSC Adv. 8(46), 25909-25919 (2018).

-. Provides evidence of a novel application for pretreatment with immunosuppressant nanoformulations to reduce chronic rejection.

79. Cardenas ME, Zhu D, Heitman J. Molecular mechanisms of immunosuppression by cyclosporine, FK506, and rapamycin. Curr. Opin. Nephrol. Hypertens. 4(6), 472-477 (1995).

80. Tocci MJ, Sigal NH. Recent advances in the mechanism of action of cyclosporine and FK506. Curr. Opin. Nephrol. Hypertens. 1(2), 236-242 (1992).

81. Pennington CA, Park JM. Sublingual tacrolimus as an alternative to oral administration for solid organ transplant recipients. Am. J. Health Syst. Pharm. 72(4), 277-284 (2015).

82. Monchaud C, Marquet P. Pharmacokinetic optimization of immunosuppressive therapy in thoracic transplantation: part II. Clin. Pharmacokinet. 48(8), 489-516 (2009).

83. Collin C, Boussaud V, Lefeuvre $S$ et al. Sublingual tacrolimus as an alternative to intravenous route in patients with thoracic transplant: a retrospective study. Transplant. Proc. 42(10), 4331-4337 (2010).

84. Park SI, Felipe CR, Pinheiro-Machado PG, Garcia R, Tedesco-Silva H Jr, Medina-Pestana JO. Circadian and time-dependent variability in tacrolimus pharmacokinetics. Fundam. Clin. Pharmacol. 21(2), 191-197 (2007).

85. Randhawa PS, Starzl TE, Demetris AJ. Tacrolimus (FK506)-associated renal pathology. Adv. Anatom. Pathol. 4(4), 265-276 (1997). 
86. Brandacher G, Lee WP, Schneeberger S. Minimizing immunosuppression in hand transplantation. Expert Rev. Clin. Immunol. 8(7), 673-683 (2012).

87. Schnider JT, Weinstock M, Plock JA et al. Site-specific immunosuppression in vascularized composite allotransplantation: prospects and potential. Clin. Dev. Immunol. 2013, 495212 (2013).

88. Xu S, Olenyuk BZ, Okamoto CT, Hamm-Alvarez SF. Targeting receptor-mediated endocytotic pathways with nanoparticles: rationale and advances. Adv. Drug Deliv. Rev. 65(1), 121-138 (2013).

89. Yu SS, Lau CM, Thomas SN et al. Size- and charge-dependent non-specific uptake of PEGylated nanoparticles by macrophages. Int. J. Nanomed. 7, 799-813 (2012).

90. Caracciolo G. Liposome-protein corona in a physiological environment: challenges and opportunities for targeted delivery of nanomedicines. Nanomedicine 11(3), 543-557 (2015).

91. Lundqvist M, Stigler J, Elia G, Lynch I, Cedervall T, Dawson KA. Nanoparticle size and surface properties determine the protein corona with possible implications for biological impacts. Proc. Natl Acad. Sci. USA 105(38), 14265-14270 (2008).

92. Neagu M, Piperigkou Z, Karamanou K et al. Protein bio-corona: critical issue in immune nanotoxicology. Arch. Toxicol. 91(3), 1031-1048 (2017).

93. Westmeier D, Stauber RH, Docter D. The concept of bio-corona in modulating the toxicity of engineered nanomaterials (ENM). Toxicol. Appl. Pharmacol. 299, 53-57 (2016).

94. Choi K, Riviere JE, Monteiro-Riviere NA. Protein corona modulation of hepatocyte uptake and molecular mechanisms of gold nanoparticle toxicity. Nanotoxicology 11(1), 64-75 (2017).

95. Nagy A, Zane A, Cole SL, Severance M, Dutta PK, Waldman WJ. Contrast of the biological activity of negatively and positively charged microwave synthesized CdSe/ZnS quantum dots. Chem. Res. Toxicol. 24(12), 2176-2188 (2011).

96. Dos Santos T, Varela J, Lynch I, Salvati A, Dawson KA. Quantitative assessment of the comparative nanoparticle-uptake efficiency of a range of cell lines. Small 7(23), 3341-3349 (2011).

97. Ferraro D, Anselmi-Tamburini U, Tredici IG, Ricci V, Sommi P. Overestimation of nanoparticles-induced DNA damage determined by the comet assay. Nanotoxicology 10(7), 861-870 (2016).

98. George JM, Magogotya M, Vetten MA, Buys AV, Gulumian M. From the cover: an investigation of the genotoxicity and interference of gold nanoparticles in commonly used in vitro mutagenicity and genotoxicity assays. Toxicol. Sci. 156(1), 149-166 (2017).

99. Pal AK, Bello D, Budhlall B, Rogers E, Milton DK. Screening for oxidative stress elicited by engineered nanomaterials: evaluation of acellular DCFH assay. Dose Response 10(3), 308-330 (2012).

100. Hofmann F, Blasche R, Kasper M, Barth K. A co-culture system with an organotypic lung slice and an immortal alveolar macrophage cell line to quantify silica-induced inflammation. PLoS ONE 10(1), e0117056 (2015).

101. Kabadi PK, Rodd AL, Simmons AE, Messier NJ, Hurt RH, Kane AB. A novel human 3D lung microtissue model for nanoparticle-induced cell-matrix alterations. Part. Fibre Toxicol. 16(1), 15 (2019).

102. Zhang M, Xu C, Jiang L, Qin J. A 3D human lung-on-a-chip model for nanotoxicity testing. Toxicol. Res. 7(6), 1048-1060 (2018).

103. Sambale F, Lavrentieva A, Stahl F et al. Three dimensional spheroid cell culture for nanoparticle safety testing. J. Biotechnol. 205, $120-129$ (2015).

104. Leite PEC, Pereira MR, Harris G et al. Suitability of 3D human brain spheroid models to distinguish toxic effects of gold and poly-lactic acid nanoparticles to assess biocompatibility for brain drug delivery. Part. Fibre Toxicol. 16(1), 22 (2019).

105. Astashkina AI, Jones CF, Thiagarajan G et al. Nanoparticle toxicity assessment using an in vitro 3-D kidney organoid culture model. Biomaterials 35(24), 6323-6331 (2014).

106. Lee J, Lilly GD, Doty RC, Podsiadlo P, Kotov NA. In vitro toxicity testing of nanoparticles in 3D cell culture. Small 5(10), 1213-1221 (2009).

107. Frohlich E. Comparison of conventional and advanced in vitro models in the toxicity testing of nanoparticles. Artif. Cell. Nanomed. Biotechnol. 46(Suppl. 2), 1091-1107 (2018).

- Discussion of next generation toxicity models and future prospects for applications in nanotoxicity.

108. Susewind J, De Souza Carvalho-Wodarz C, Repnik U et al. A 3D co-culture of three human cell lines to model the inflamed intestinal mucosa for safety testing of nanomaterials. Nanotoxicology 10(1), 53-62 (2016).

109. Walczak AP, Kramer E, Hendriksen PJ et al. Translocation of differently sized and charged polystyrene nanoparticles in in vitro intestinal cell models of increasing complexity. Nanotoxicology 9(4), 453-461 (2015).

110. Chia SL, Tay CY, Setyawati MI, Leong DT. Biomimicry 3D gastrointestinal spheroid platform for the assessment of toxicity and inflammatory effects of zinc oxide nanoparticles. Small 11(6), 702-712 (2015).

111. Ude VC, Brown DM, Stone V, Johnston HJ. Using 3D gastrointestinal tract in vitro models with microfold cells and mucus secreting ability to assess the hazard of copper oxide nanomaterials. J. Nanobiotechnol. 17(1), 70 (2019). 